\title{
Development of tunnel lining inspection method by the elastic-wave transfer function $\operatorname{method(ETFuM)~}$
}

\author{
Hiroyuki Morishima \\ Hiroo Koyama \\ Yakichi Higo \\ Satomi Tunoda \\ Yukinori Kiuchi
}

\author{
East Japan Railway Company \\ HEast Japan Railway Company \\ P\&I Lab. Tokyo Institute of Technology \\ Taisei Corporation \\ Nakabohtec corrosion protecting CO.,LTD
}

\begin{abstract}
Japanese railway tunnels are currently inspected by using destructive testing. The inspection procedure consists of three important inspecting items, such as lining thickness, voids between a lining and the natural ground, and compressive strength. This method has difficulties in measuring accuracy, cost and Efficiency of inspection.

In order to solve these problems, we have developed The Elastic-wave Transfer Function Method (ETFuM) an efficient non-destructive inspection technique which can be applied to the measurement of the above mentioned three items .

The results of a fundamental experiment and a site test on ETFuM demonstrated the high feasibility of the practical application of this method to the railway tunnel inspection in the near future.
\end{abstract}

1

Introduction

There are several methodological streams for non-destructive inspecting of tunnel structures, such as electromagnetic wave method, thermo-graphy method and ultrasonic method. Some problems are pointed out in practical use of these current methods:(1)Electromagnetic wave method can be used for measurement of lining thickness, and voids between a lining and the natural ground only under the circumstance that neither steel supports are located close together in the lining nor the lining consists of reinforced concrete. (2) Thermography method is not suitable for tunnel lining inspection, because temperature differences on the structure surface, which are obscure in tunnels, are essential for the detection of inner voids by use of this method. (3) Ultrasonic method is not reliable enough for inspection of tunnel linings, because ultrasonic waves attenuate much more quickly in concrete than in metallic materials for which the method is suitable.

The ETFuM(Elastic Wave Transfer-function Method) which we developed as a nondestructive inspection method for tunnel lining makes it possible to inspect tunnels in three important items : lining thickness, voids between a lining and the natural ground and compressive strength.

Theory of the method and experimental results of test in which this method was applied to both fundamental models and a real tunnel are described in this paper. 
2

2.1 Transfer function

An explanation is presented of the principle by which the transfer function of the ultrasonic wave is measured using ETFuM. A schematic diagram is showing the transfer of an ultrasonic wave through the specimen including object part, $\alpha$, as shown in Fig.2-3 [1].The transfer function of the system, HA, is expressed as follows,

$$
\begin{aligned}
H_{A n} & =G_{A n} / F_{n} \\
& =S_{n} \beta_{1 n} h_{1 n} \alpha_{n} \quad h_{2 n} M_{n}
\end{aligned}
$$

$M n$ and $S n$ are the received and transmitted transducer sensitivity, $\beta$ is transfer function expressing coupling condition between transducer and specimen. Where $n$ indicates specimen or measurement number.

Fig.2-2 shows a simple example on the transfer function applied to ETFuM.In Fig.2-2 (a) is perfect tuning fork, but (b) and (c) include defect, and (d) include a hair crack.

is very easy to estimate the defect of tuning fork by hearing the sound. The $f(t)$ in Fig.2-1 corresponds to the action of striking the tuning fork with a hammer in Fig.2-1 and $g(t)$ is the resulting sound. Where as $h(t)$ is the transfer function of the fork, we only want to obtain information on the specimen from $H_{\lambda}$, such as the tuning fork.

So we need separate the object information, $\alpha \mathbf{n}$, from the characteristics of the transducers, $S n$ and $M n$, the two parts of joint, $h_{1}$ and $h_{2}$, and the coupling condition between the transducers and testpieces, $\beta_{1 n}$ and $\beta_{2 n}$. We will use test pieces that are acoustically equivalent to $h_{1}$ and $h_{2}$ and the same transducers, shown here as $S n$ and $M n$. In addition, we have developed a method of coupling the transducers to the test specimen so as to obtain good acoustic reproducibility. The transfer function of $H_{12}$, including a new part, $\alpha$ 2 , is diffident in the same way as $H_{11}$, which includes the standard joint, $\alpha_{1}$. Then the ratio of $H_{A_{2}}$ and $H_{A 1}$ is as follows,

$$
\begin{aligned}
& H_{A 1} / H_{A 2}=\left(G_{A 1} / F_{1}\right) /\left(G_{A 2} / F_{2}\right) \\
& =\left(\begin{array}{lllllll}
S_{1} & \beta_{11} & h_{11} & \alpha_{1} & h_{21} & \beta_{21} & M_{1}
\end{array}\right) /\left(\begin{array}{lllllll}
S_{1} & \beta_{12} & h_{12} & \alpha_{2} & h_{22} & \beta_{22} & M_{2}
\end{array}\right)
\end{aligned}
$$

As all the components of the transfer function that reflect the characteristics of the transducers $S$ and $M$, the test pieces $h_{1}$ and $h_{2}$, and the coupling conditions $\beta_{1}$ and $\beta_{2}$ are canceled out, only the information on the void between lining and natural ground is left as follows,

$$
H_{A 1} / H_{A 2}=\left(G_{A 1} / F_{1}\right) /\left(G_{A 2} / F_{2}\right)=\alpha_{1} / \alpha_{2}
$$

Also, since the signal source $F$ provides good reproducibility, the ratio of the output signal $G_{A 1}$ to $G_{\mathcal{A}}$ directly explains the difference in the condition of the void between lining and natural ground as follows, 


$$
\alpha_{1} / \alpha_{2}=G_{A 1} / G_{A 2}
$$

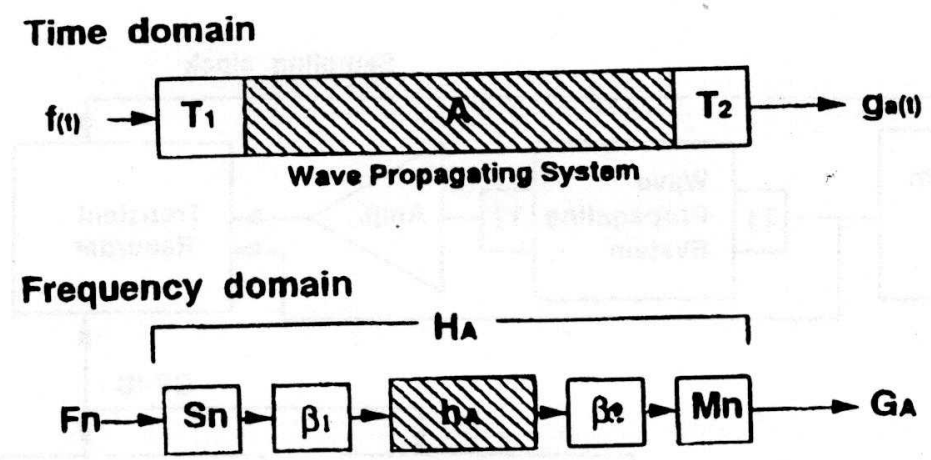

Figure 2-1. The transfer function of the system, $H_{\Lambda}$, including object part, $\alpha . h_{1}$ and $h_{2}$ are the test pieces, and $\beta_{1}$ and $\beta_{2}$ are the coupling conditions between sensor and specimen.

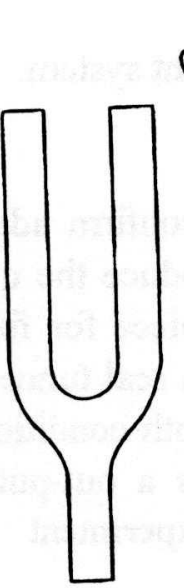

(a)

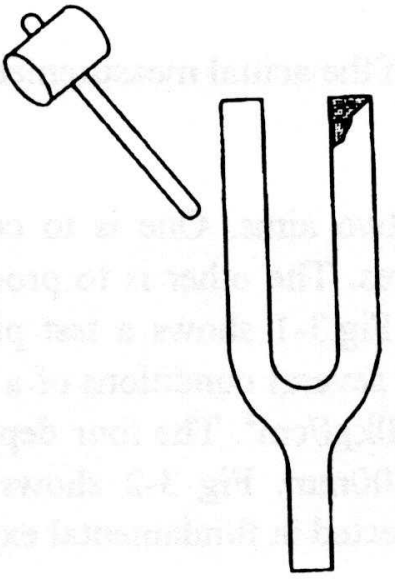

(b)

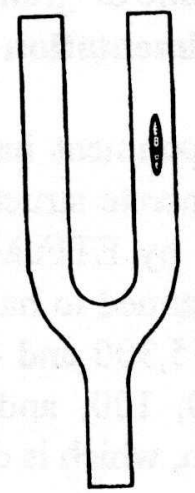

(c)

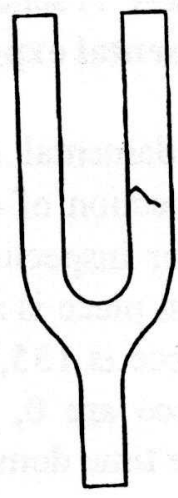

(d)

Figure 2-2 A simple example on the transfer function applied to ETFuM. (a) is a perfect tuning fork, (b) and (c)are those include defects, and (d) is with a hair crack.

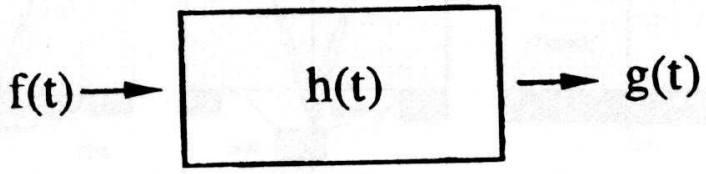

Figure 2-3 The relation between the input signal, $f(t)$, and the output signal, $g(t)$. The $h(t)$ is transfer function of object specimen. 


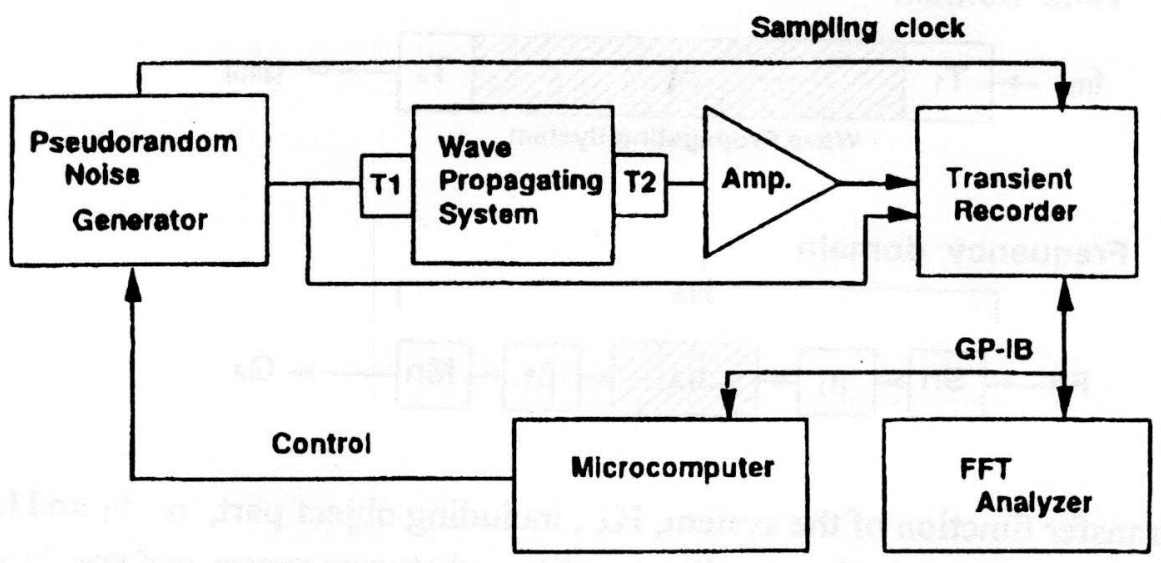

Figure2-4 A schematic diagram of the actual measurement system.

3 Fundamental experimentation

\subsection{Outline}

Our fundamental experiment has two aims. One is to confirm adaptation of ETFuM to the inspection of concrete structures. The other is to produce the quantitative calibration curve for inspection by ETFuM. Fig.3-1 shows a test piece for fundamental experiment. The test piece is assumed to have several conditions of a real tunnel. Strength condition of test piece is $135,255,300$ and $400 \mathrm{kgf} / \mathrm{cm}^{2}$. The four depth conditions of void behind the test piece are $0,40,100$, and $200 \mathrm{~mm}$. Fig 3-2 shows a out-put signal of accelerometer in the time domain, which is detected in fundamental experiment

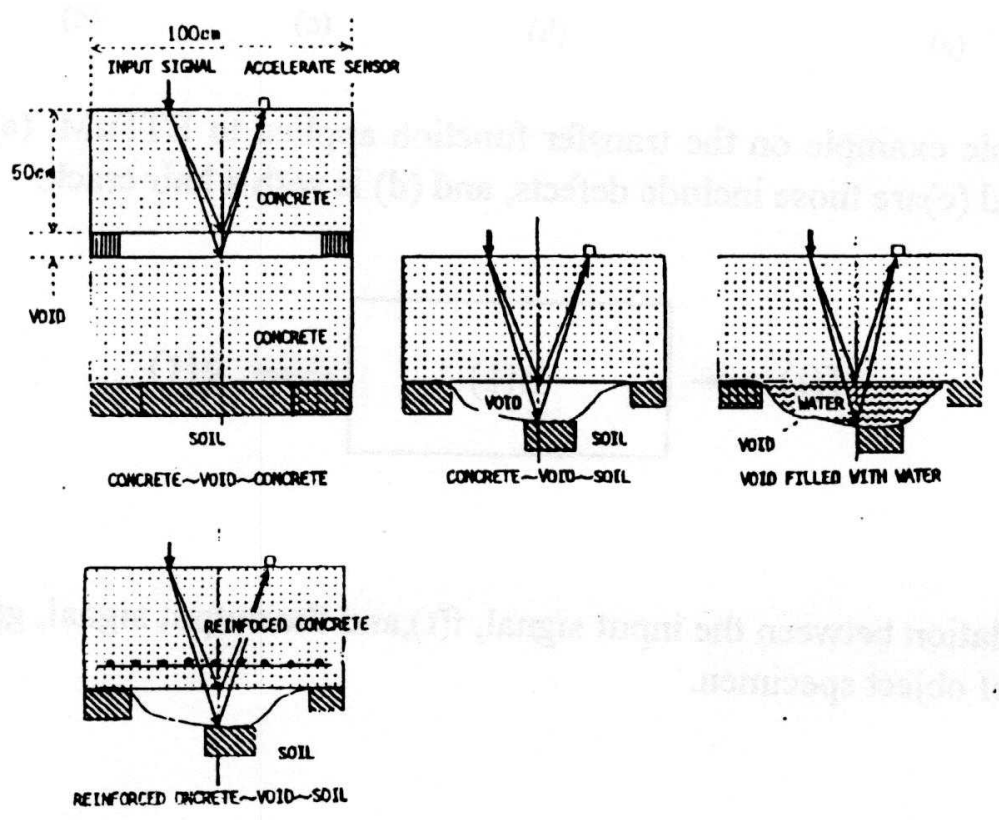

Figure. 3-1 The test pieces for fundamental experiment. 


\subsection{Principle for inspection of the tunnel lining}

The attenuation of power spectrum in less than $5 \mathrm{kH}$ for output signals measured by accelerometers of which resonance frequency is near $50 \mathrm{kH}$ was clearly stronger in case that voids consist in the test piece than the case that the test piece contains no voids in it.

Also, since the signal source $\mathrm{F}$ provides good reproducibility, the ratio of the output signals $G_{\boldsymbol{A} 1}$ to $G_{\boldsymbol{A}^{2}}$ directly explains the difference in the void condition. In other words, when we measure the level of output signal Y (Fig. 3-2), we can inspect voids behind lining. If we measure the lapse of first wave of minus height $X$ (Fig.3-2), and the lapse of first wave of plus height X (Fig. 3-2), we can estimate the compressive strength of concrete linings. And, if we measure the propagating lapse time of the elastic wave and the propagating velocity of the elastic wave, we can calculate the lining thickness. This method can be applied to three items by measuring output signals of one shot of elastic wave.

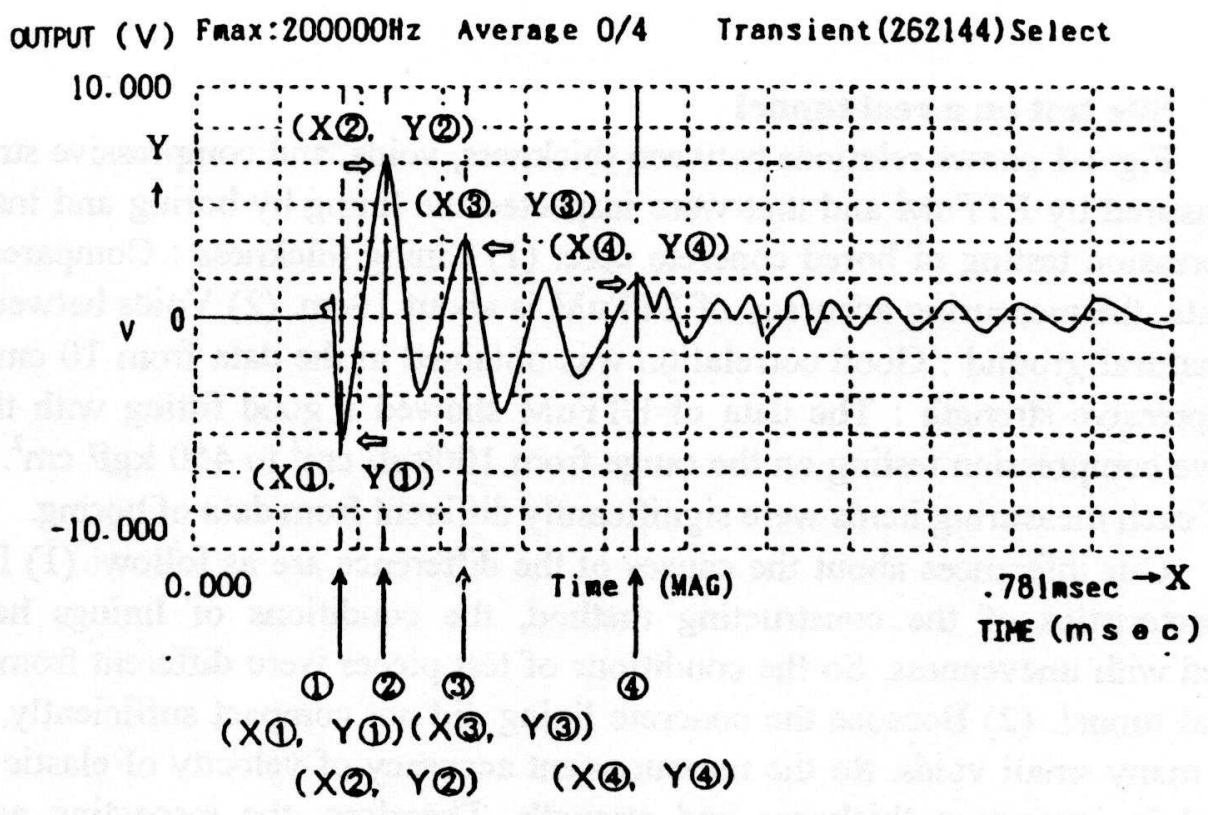

Figure 3-2 A output signal of accelerometer in the time domain.

\subsection{Site test on a real railway tumnel}

This site test has two aims. One is to gather data from a real tunnel by ETFuM, the other is to compare the data from ETFuM with the data obtained by boring test or by destructive conversion testing.

The tunnel tested was planned for single track of electrified railway. And, lining thickness was planned for $400 \mathrm{~mm}$. Compressive strength of the lining was planned for $160 \mathrm{kgf} / \mathrm{cm}^{2}$.

We chose 4 cross sections in the tunnel for this site test, and each cross section 
has 5 measuring points at the tunnel arch.

4 tunnel

4.1 Fundamental experiment

If the fundamental experiment performs strictly by the principle of ETFuM, the measuring data by ETFuM have to be adjusted in accordance with physical characteristics of the transducers. But, in this case, we did not need to adjust the data, because the input signal source provided good reproducibility. (2)We could carried out the three items of tunnel inspection by measuring the components of signals in frequency domain. These items are Lining thickness, Voids between lining and natural ground and Compressive strength. (3) This inspecting method was useful for not only concrete but reinforced concrete.

\subsection{Site test on a real tunnel}

Fig 4-1 shows relations between thickness, voids, and compressive strength that were measured by ETFuM and that were inspected the lining by boring and inspected by the compression testing of bored concrete core. (1) Lining thickness : Compared with the boring data, the measuring accuracy of ETFuM is about $10 \mathrm{~cm}$. (2) Voids between a lining and the natural ground: Good correlation was obtained in the data from $10 \mathrm{~cm}$ to $60 \mathrm{~cm}$. (3) Compressive strength : The data of ETFuM showed a good fitting with the data of destructive compression testing on the range from $100 \mathrm{kgf} / \mathrm{cm}^{2}$ to $450 \mathrm{kgf} / \mathrm{cm}^{2}$. But some datum of each measuring items were significantly different from data of boring.

Our inferences about the causes of the difference are as follow: (1) Because of the characteristics of the constructing method, the conditions of linings have been completed with unevenness. So the conditions of test pieces were different from the lining of the real tunnel. (2) Because the concrete lining did not compact sufficiently, the lining includes many small voids. So the measurement accuracy of velocity of elastic wave was concerned in inspecting thickness and strength. Therefore, the inspecting accuracy of thickness and the inspecting accuracy of strength were affected by the measurement accuracy of velocity of elastic wave. (3) Because the reflecting elastic wave from small voids inside lining or the reflecting elastic wave from some obstacles,(Probably timber supports remained in the lining), the inspecting accuracy was affected.

Considering these problems, We need following some improvements. (1) When the velocity of surface elastic wave is measured in infinitesimal time, the accuracy must be improved.(2) Out put signals need to be analyzed with scrupulous attention in order to select some affected output signals from all of them. If these improvements are performed, this method will be most useful non-destructive method for tunnel inspection. 

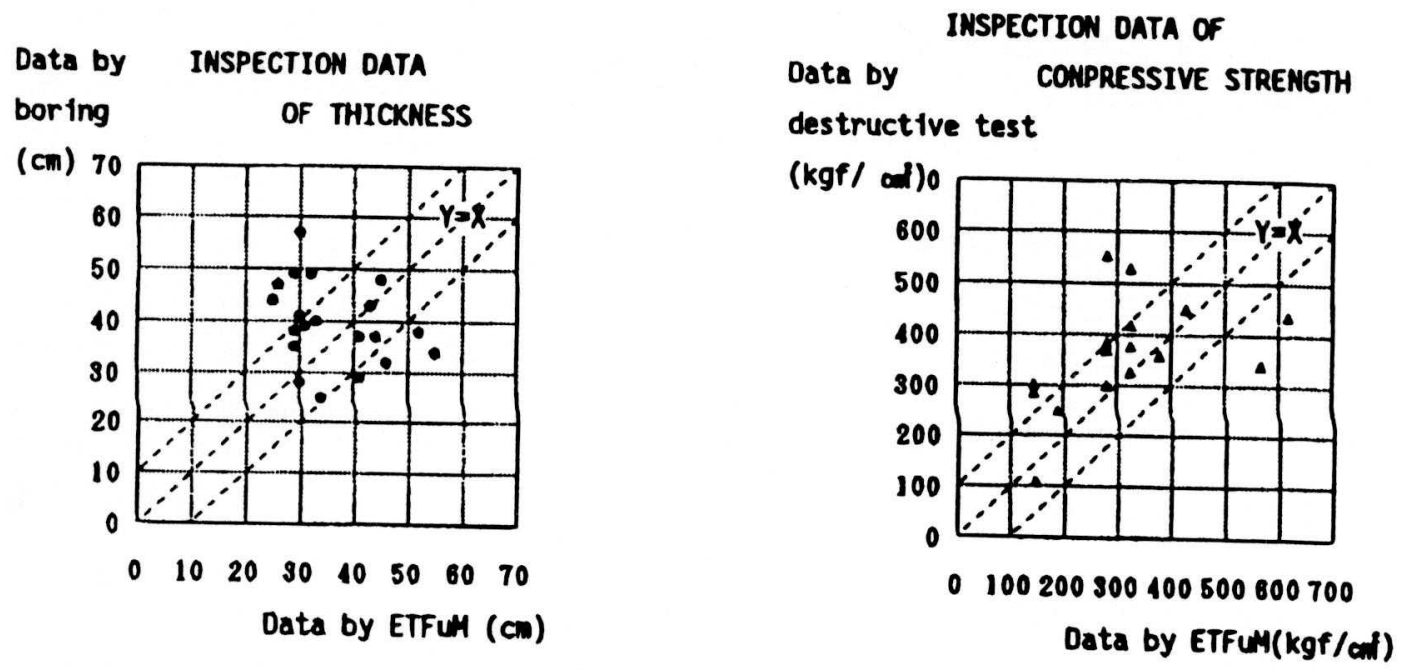

Data by INSPECTION DATA

boring Of volos

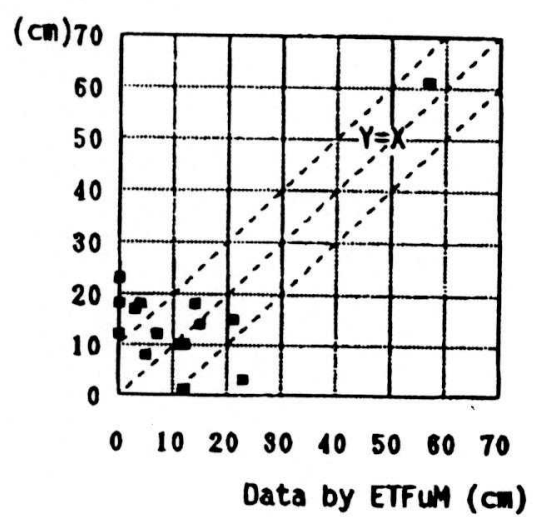

Figure 4-1 The relation between datum of each items by ETFuM and inspection datum by boring or by destructive compression testing

[1]Higo,Y., Kazama,S., and Nunomura,S.,"Non-destructive evaluation of $m$ aerials and structures by the elastic-wave transfer function method Engineering, A146(1991)pp327-333

Materials Science and [2]Tsunoda,S., Kiuchi,Y., and Higo,Y.,"A Qualitative Method For Deterring Decohesion Between A Heat Resistant Material Adhered Proc.of Int. Conf. on Monitoring and Adhered Steel Reaction Tower by ETFuM Structures(Firenze, Italy), (1992)p p253-258 